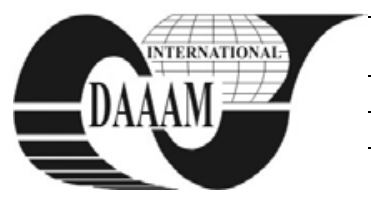

\title{
NUMERICAL SIMULATION OF THE LASER BEAM DRILLING PROCESS
}

\author{
BEGIC, D[erzija]; BIJELONJA, I[zet]; KULENOVIC, M[alik] \& CEKIC, A[hmet]
}

\begin{abstract}
A numerical model for the prediction of axisymmetric temperature distribution and the shape of hole in tungsten alloy during laser drilling has been developed using a finite volume method (FVM). The energy balance equation in integral form is used for a description of the temperature field. Verification of the application of finite volume method is performed by comparing the results of numerical calculations with experimental data. It was found good agreement of results and shown that the finite volume method can be an effective tool to predict the shape of hole and size of the heat affected zone (HAZ) during the laser drilling.
\end{abstract}

Key words: laser, drilling, tungsten alloy, finite volume method

\section{INTRODUCTION}

Laser beam machining is one of the most widely used thermal energy based non-contact type advance machining process which can be applied for almost whole range of materials. Thermal models for investigation may be divided into two main categories: models with detailed treatment of thermal conduction and models where details of phase transition (melting, vaporization) are considered. (Junke \& Wang, 2008) have used FEM software Ansys to analyse the temperature and thermal stress distributions in laser cutting of glass. (Kim, 2004) developed an unsteady convective heat transfer model using BEM considering moving continuous Gaussian laser beam for the prediction of groove shape, groove depth, temperature and flux distribution. A FEM based unsteady heat transfer model for the prediction of amount of material removal and groove smoothness during evaporative machining with a Gaussian wave pulsed laser has also developed (Kim \& Zhang, 2001).

The objective of this paper is to develop a $2 \mathrm{D}$ numerical model based on the finite volume method in order to predict the temperature distribution and the shape of hole during laser drilling of tungsten alloy with a thickness of $1 \mathrm{~mm}$. Considering the heat input from laser beam as a fixed heat source, an unsteady heat transfer equation was considered that deals with the drilling process using a continuous Gaussian laser beam.

\section{MATHEMATICAL FORMULATION}

The diagram of the laser processing is illustrated in Fig.1.

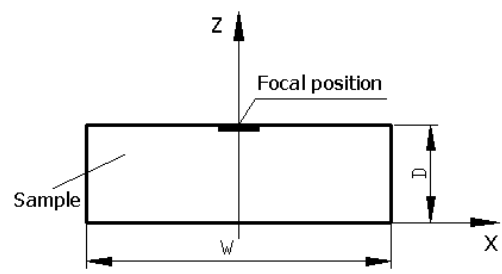

Fig. 1 . The diagram of the laser processing

The mathematical model for prediction of the temperature distribution during laser drilling can be written as follows:

$$
\begin{gathered}
\frac{\partial}{\partial t} \int_{V} \rho c T d V=\int_{S} \mathbf{q} \cdot \mathbf{n} d S+\int_{V} h_{s} d V \\
T(t)=T_{o} \text { at } t=0,
\end{gathered}
$$

where $V$ is volume of sample bounded by the surface $S, \mathbf{q}$ is the heat flux vector, $\mathbf{n}$ is the unit outward surface normal, $h_{\mathrm{s}}$ is the sink of heat, $c$ and $\rho$ are the specific heat and the density, respectively. $T_{0}$ denotes the initial temperature of sample.

The relation between the heat flux and temperature gradient is given by Fourier's law:

$$
\mathbf{q}=k \operatorname{grad} T,
$$

where $k$ is the thermal conductivity.

The boundary condition on the surface which is subjected to the laser beam is obtained from the balance of energy on the surface as:

$$
-k \frac{\partial T}{\partial z}=h\left(T_{\infty}-T_{s}\right)+\varepsilon \sigma\left(T_{\infty}^{4}-T_{s}^{4}\right)+\alpha I(r, t)
$$

and boundary conditions on the other boundaries are:

$$
\begin{gathered}
-k \frac{\partial T}{\partial n}=h\left(T_{\infty}-T\right)+\varepsilon \sigma\left(T_{\infty}^{4}-T^{4}\right) \\
\text { and at } z=0 ; \frac{\partial T}{\partial x}=0
\end{gathered}
$$

where, $h$ is the convection heat transfer coefficient, $T_{s}$ denotes the temperature of the heated zone surface and $T_{n}$ denotes the temperature of the area without laser heating, $\sigma$ is the StefanBoltzmann constant, $\alpha$ is the laser absorptivity, $I(r, t)$ is the intensity of the laser beam, and $T_{\infty}$ is the environment temperature.

\section{NUMERICAL METHOD}

The governing equation, together with non-linear boundary conditions, applied to the two-dimensional drilling geometry is solved using finite volume method. In order to obtain the discrete counterparts of equations (1), (4) and (5), the time is discretized into an arbitrary number of time steps of the size $\delta t$ and the solution domain is subdivided into a finite number of contiguous quadrilateral controls volumes (CV) or cells of volume $V$ bounded by the surface $S$. The inertial term is approximated using the backward differencing, and volume integrals are estimated using midpoint rule. The set of linearized equations for each variable is solved at each time step by iteration. A fully implicit scheme is used for the time dependent terms.

Particular attention has been focused on modelling of boundary conditions in the area of interaction between laser beam and work piece, and changing boundaries of spatial 
domain of integration. Boundary conditions are defined by the heat exchange between the work piece and environment, including all three types of heat transfer: conduction, convection and radiation. Spatial domain of integration in the process of drilling is variable due to the removal of material from the machining zone, which makes the problem nonlinear. A detailed description of the numerical algorithm applied in this work is given in (Begic et al., 2008).

\section{RESULTS AND DISCUSSION}

For numerical analysis used a sample of size $1.5 \mathrm{x} 1 \mathrm{~mm}$. The initial temperature of sample is the same as the environment temperature. The z-axis, Fig. 1, is the symmetry axis of the tungsten alloy plate.

The thermophysical properties of tungsten alloy, process parameters, and parameters important for the numerical calculation are shown in Table 1.

\begin{tabular}{|l|l|l|}
\hline & Properties & Values \\
\hline Tungsten alloy & $T_{L}$ & $3100{ }^{\circ} \mathrm{C}$ \\
\hline & $H_{L}$ & $250 \mathrm{~kJ} \mathrm{~kg}^{-1}$ \\
\hline & $\alpha$ & 0.86 \\
\hline & $\rho$ & $17600 \mathrm{~kg} \mathrm{~m}^{-3}$ \\
\hline Other parameters & $\sigma$ & $5.67 \cdot 10^{-8} \mathrm{Wm}^{-2} \mathrm{~K}^{-4}$ \\
\hline & $H$ & $25 \mathrm{~W} \mathrm{~m}^{-2} \mathrm{~K}^{-1}$ \\
\hline & $t_{\text {tot }}$ & $8 \mathrm{~ms}$ \\
\hline & $P$ & $1000 \mathrm{~W}$ \\
\hline & $r$ & $0.105 \mathrm{~mm}$ \\
\hline
\end{tabular}

Tab. 1. Thermophysical properties of sample and other parameters

The emission coefficient $\varepsilon$, the thermal conductivity $k$, and the specific heat $c$ are considered as temperature-dependent parameters for numerical calculation (Lassner and Schubert, 1999).

In Figs. 2 and 3, the temperature distribution is shown for the finest mesh $8664 \mathrm{CVs}$ and the time step $0.05 \mathrm{~ms}$ during laser drilling at the times $0.5 \mathrm{~ms}$ and $2 \mathrm{~ms}$, respectively.

A quantitative estimate of numerical results was performed by the experiment, as shown in Figs. 4 and 5.

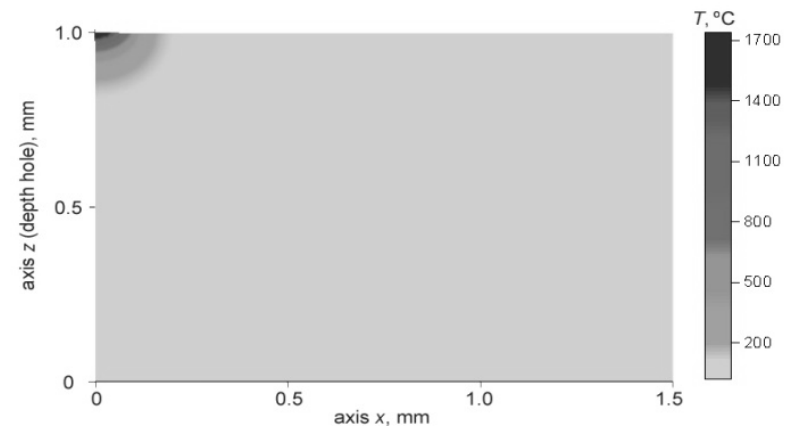

Fig. 2. The temperature distribution at the time $0.5 \mathrm{~ms}$

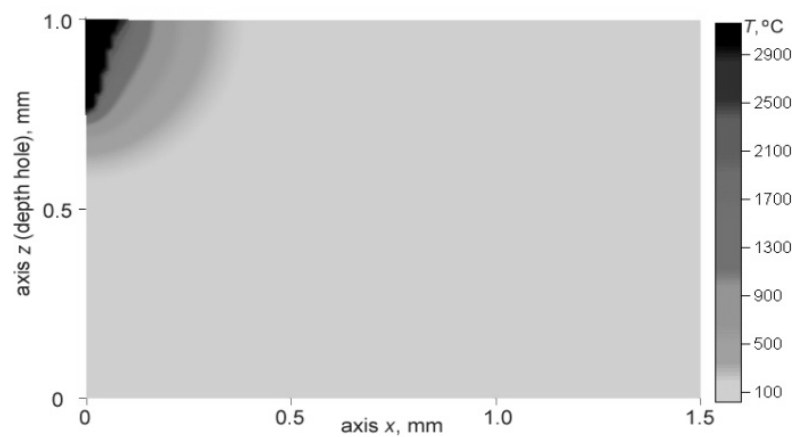

Fig. 3. The temperature distribution and the hole profile at the time $2 \mathrm{~ms}$

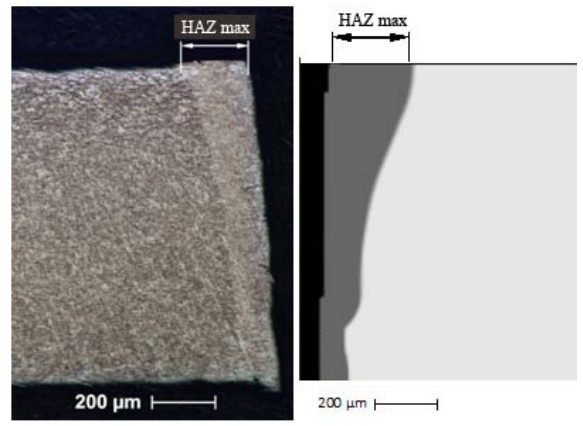

Fig. 4. The size of HAZ: experiment (left), FVM (right)

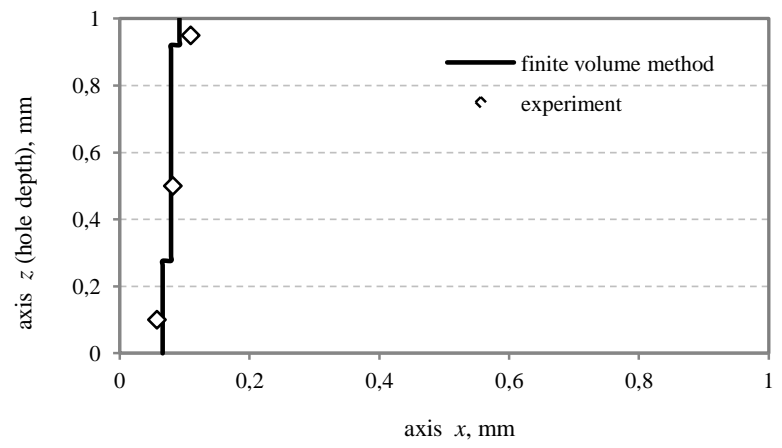

Fig. 5. The shape of hole in laser drilling process

\section{CONCLUSION}

A finite volume method based on the formulation discussed above is developed to study the time-dependent material removal of a sample that is subjected to a continuous laser beam of constant power. The obtained numerical results yield the qualitative good results. Verification method, which is also presented in this paper, was made by comparing the numerical results obtained with the experimental results. Comparison was made using experimental results that are obtained during $\mathrm{CO}_{2}$ laser drilling of tungsten alloy plate with a thickness of $1 \mathrm{~mm}$. In metallographic samples was measured the size of HAZ and the diameter of the hole, which was very suitable for testing the accuracy of the results obtained by calculation. Good agreement of numerical and experimental results was found.

Also, in the future work, the numerical analysis of 3D laser drilling and cutting process using finite volume method will be considered.

\section{REFERENCES}

Begic, D.; Bijelonja, I.; Kulenovic, M. \& Cekic, A. (2008). Transient evaporative laser cutting with finite volume method. Proceedings of the $19^{\text {th }}$ International DAAAM Symposium, Katalinic, B., pp. 0079-0080, ISBN 978-3901509-68-1, Published by DAAAM International, Vienna

Junke, J. \& Wang, X. (2008). A numerical simulation of machining glass by dual CO2-laser beams. Optics \& Laser Technology, 40, 2, (March 2008) page numbers (297-301), ISSN 0030-3992

Kim, M.J. (2004). Transient evaporative laser cutting with moving laser by boundary element method. Applied Mathematical Modelling, 28, 10, (October 2004) page numbers (891-910), ISSN 0307-904X

Kim, M.J. \& Zhang, J. (2001). Finite element analysis of evaporative cutting with a moving high energy pulsed laser. Applied Mathematical Modelling, 25, 3, (January-February 2001) page numbers (203-220), ISSN 0307-904

Lassner, E. \& Schubert, W.D. (1999). Tungsten - Properties, Chemistry, Technology of Element, Alloys, and Chemical Compounds, Kluwer Academic, New York, 1999. 